\title{
ANÁLISES QUÍMICAS E DENSIDADE BÁSICA DA MADEIRA DE RAIZ, FUSTE E GALHO DE BARBATIMÃO [(Stryphnodendron adstringens) Coville] DE BIOMA CERRADO
}

\author{
Selma Lopes Goulart ${ }^{1}$, Fábio Akira Mori², Alessandra de Oliveira Ribeiro ${ }^{3}$, Allan Motta Couto ${ }^{4}$, \\ Marina Donária Chaves Arantes ${ }^{5}$, Lourival Marin Mendes $^{6}$
}

(recebido: 13 de janeiro de 2010; aceito: 28 de outubro de 2011)

\begin{abstract}
RESUMO: A região do cerrado tem se mostrado como importante provedora na crescente demanda por bens de consumo de origem vegetal, animal ou agroindustrial. No entanto, ainda são insuficientes os estudos relativos à vegetação desse bioma. Tal fato tem causado dificuldades na utilização dessas espécies, seja como opção de uso econômico, ou como forma de recomposição e recuperação de áreas devastadas. Em razão da carência de informações referentes às espécies do cerrado, este estudo foi realizado com o objetivo de coletar informações sobre a constituição química e a densidade básica da madeira de raiz, fuste e galho de Stryphnodendron adstringens (barbatimão). Para tanto, utilizaram-se materiais coletados na raiz e ao longo do fuste e galho de três espécimes de barbatimão. Verificaram-se tendências de incremento dos teores de holocelulose no sentido da raiz para o fuste e galho. O contrário aconteceu com o teor de lignina e extrativos. O teor de cinzas teve seu menor valor na posição referente ao fuste. As densidades básicas entre raiz, fuste e galho não se diferenciaram estatisticamente.
\end{abstract}

Palavras-chave: Lenho, análise química, árvore nativa, planta.

\section{CHEMICAL ANALYSES AND BASIC WOOD DENSITY IN THE ROOT, STEM AND BRANCH PORTIONS OF BARBATIMÃO [(Stryphnodendron adstringens) Coville] FROM THE CERRADO BIOME}

\begin{abstract}
The Cerrado region has been a major provider to meet the growing demand of vegetal, animal or agribusiness consumer goods. However, studies on the vegetation of this biome are still incipient and that has been preventing use of Cerrado species, whether for economic purposes or as a way of restoring and recovering devastated areas. Due to lack of information concerning species of the Cerrado biome, this study was conducted in an attempt to gather information about the chemical constitution and basic wood density of the root, stem and branch portions of Stryphnodendron adstringens, also known as 'barbatimão'. To that end, material was collected from the root and along stem and branch portions of three specimens of barbatimão. An increasing tendency was observed in holocellulose contents in the root-to-stem and root-to-branch direction. The opposite occurred with lignin and extractive contents. The lowest ash content was found in the stem portion. Values of basic density did not differ statistically between the root, stem and branch portions.
\end{abstract}

Key words: Wood, chemical analysis, native tree, plant.

\section{INTRODUÇÃO}

A região do cerrado, antes erroneamente considerada como uma área pobre, tem se mostrado, apesar das limitações químicas e físicas de seus solos, como importante provedora na crescente demanda por bens de consumo de origem vegetal, animal ou agroindustrial. No entanto, ainda são insuficientes os estudos relativos às plantas nativas. Causando dificuldades na utilização das espécies florestais, tanto para seu uso econômico, ou para recomposição e recuperação de áreas devastadas (SILVA et al., 2004). As espécies nativas, em sua maioria, apresentam possibilidade de múltiplos usos, além de reunirem características favoráveis de adaptação às condições do ambiente, mas, para isso, é preciso conhecimento de seu potencial tecnológico.

${ }^{1}$ Bióloga, Pós-doutoranda em Recursos Florestais e Engenharia Florestal - Departamento de Ciências Florestais/DCF - Universidade Federal de Lavras/UFLA - Cx. P. 3037 - 37200-000 - Lavras, MG - lopesgoulart@yahoo.com.br

${ }^{2}$ Engenheiro Florestal, Professor Dr. em Ciências Florestais - Departamento de Ciências Florestais/DCF - Universidade Federal de Lavras/UFLA Cx. P. 3037 - 37200-000 - Lavras, MG - morif@dcf.ufla.br

${ }^{3}$ Bióloga, Doutoranda em Ciência e Tecnologia da Madeira - Departamento de Ciências Florestais/DCF - Universidade Federal de Lavras/UFLA Cx. P. 3037 - 37200-000 - Lavras, MG - alebioribeiro@gmail.com

${ }^{4}$ Engenheiro Florestal, Doutorando em Ciência e Tecnologia da Madeira - Departamento de Ciências Florestais/DCF - Universidade Federal de Lavras/UFLA - Cx. P. 3037 - 37200-000 - Lavras, MG - allan_floresta@yahoo.com.br

${ }^{5}$ Engenheira Florestal, Professora Dra. em Ciência e Tecnologia da Madeira - Centro de Ciências Agrárias CCA/UFES - Universidade Federal do Espírito Santo/UFES - 29.500-000 - Alegre, ES - mdonariac@hotmail.com

${ }^{6}$ Engenheiro Florestal, Professor Dr. em Engenharia Florestal - Departamento de Ciências Florestais/DCF - Universidade Federal de Lavras/UFLACx. P. 3037 - 37200-000 - Lavras, MG - lourival@dcf.ufla.br

Cerne, Lavras, v. 18, n. 1, p. 59-66, jan./mar. 2012 
Ao tratar-se de estudos da qualidade da madeira, principalmente de suas propriedades físicas e químicas para espécies nativas, especialmente as do cerrado, observamos poucos trabalhos como os de Marchesan et al. (2006), caracterização física, química e anatômicas da madeira de Ocotea porosa (imbuia) e Byrsonima orbignyana, (canjiqueira) com característica exclusiva da floresta Ombrófila Mista Montana e, de campos-cerrados, respectivamente; Mattos et al. (1999) caracterização física, química e anatômica da madeira de Terminalia ivorensis, espécie procedente de florestas densas e de transição; Mendes et al. (2002), anatomia e características físicoquímicas da madeira de Zanthoxylum tingoassuiba, Silva et al. (2009), anatomia e densidade básica da madeira de Caesalpinia pyramidalis (Fabaceae), espécie endêmica da caatinga do Nordeste do Brasil.

De acordo com Panshin e Zeeuw (1980), a densidade é uma propriedade física importante da madeira, pois é um parâmetro que pode afetar outras de suas propriedades. Segundo Chimello (1980), a densidade da madeira é considerada a propriedade física mais importante, relacionando-se com outras propriedades e a sua utilização, assim, madeira de maior densidade corresponde, em geral, a uma maior resistência mecânica e alto valor energético, isso, porque a densidade está diretamente relacionada à quantidade de celulose que a constitui. Paula (1993) afirma que quanto mais alta for à massa específica (densidade) da madeira, maior será o rendimento de energia em benefício do maior teor de celulose e lignina.

Já, referindo-se às propriedades químicas da madeira, segundo Metcalfe e Chalk (1983), os estudos das estruturas químico-anatômicas do xilema secundário, além de fornecerem dados para subsidiar o seu uso, gera relações entre famílias botânicas e o estabelecimento de afinidades entre espécies ou grupos específicos. Lepage et al. (1986), comentam que quanto à sua composição química, a madeira pode ser definida como um polímero biológico tridimensional, composto por uma rede interconectada de celulose, hemicelulose e lignina, além de uma porção minoritária de extrativos e componentes inorgânicos, estes últimos, geralmente considerados componentes secundários. Segundo Klock et al. (2005), a celulose é o principal componente da madeira, chegando a constituir de $40 \%$ a $50 \%$ de todas as plantas. Trugilho et al. (2003) afirmam que para as espécies Eucalyptus grandis e Eucalyptus saligna, os valores médios de extrativos totais, de lignina e de holocelulose apresentam-se na ordem de $6,71,31,77$ e $61,52 \%$, respectivamente.

Cerne, Lavras, v. 18, n. 1, p. 59-66, jan./mar. 2012
Lignina, de acordo com (ROWELL, 2005), são estruturas amorfas, de extrema complexidade, possuindo, predominantemente, unidades poliméricas de fenilpropano. Klock et al. (2005), comentam, ainda, que a estrutura da lignina pode relacionar-se, entre outros, com o aumento da rigidez da parede celular, a redução da permeabilidade da madeira e a proteção da madeira contra agentes xilófagos, em razão da sua natureza fenólica. Em estudos físico-químicos da madeira de Eucalyptus saligna realizados por Trugilho et al. (1996), o teor de lignina apresentou correlação negativa com a massa volumétrica básica e o teor de holocelulose, mostrando que quanto menor for o teor de lignina maior será o teor de holocelulose e menor será a massa volumétrica básica da madeira. Severo et al. (2006), concluíram, em seu trabalho com Eucalyptus citriodora, que a percentagem de lignina foi sempre superior no lenho juvenil e a percentagem de holocelulose foi sempre menor no lenho juvenil.

Buchanan (1981), Morais et al. (2005) e Oliveira et al. (2005) comentam que os extrativos têm como característica, não fazer parte da estrutura da parede celular, considerados constituintes secundários, possui baixa massa molecular, são solúveis em água ou em solventes orgânicos neutros e estão presentes principalmente na casca. Apresenta-se em pequenas proporções, mas mesmo assim, estão entre os principais responsáveis pela resistência da madeira ao ataque de fungos e insetos (BUCHANAN, 1981; OLIVEIRA et al., 2005). Geralmente, são representados pelos óleos essenciais, resinas, taninos, graxas e pigmentos (MORAIS et al., 2005). Trugilho et al. (1996), estudando a madeira de Eucalyptus saligna concluiram que existe uma tendência de estabilização nos valores de extrativos totais, com a idade. Severo et al. (2006) comentam que o Eucalyptus citriodora, no lenho adulto há um decréscimo de extrativos totais com a altura e, segundo os autores, a percentagem de extrativos totais foi menor no lenho juvenil da primeira à quarta tora e nas restantes ocorreu comportamento contrário.

Os principais íons minerais normalmente encontrados em cinzas de madeira, segundo Freddo (1997) são: cálcio, magnésio, potássio, baixas quantidades de sódio, manganês, alumínio, ferro, além de radicais como silicatos, carbonatos, cloretos, sulfatos. Existem também traços de outros elementos como zinco, cobre e cromo, dentre outros. A quantidade desses elementos nas árvores varia com a espécie, a disponibilidade no 
solo, a necessidade individual e a época do ano. Esses componentes inorgânicos de acordo (BUCHANAM, 1981), proporcionam à madeira uma maior resistência a organismos como os crustáceos e moluscos. Os componentes inorgânicos, geralmente, são representados por cristais de oxalatos de cálcio ou potássio e sílica, e a proporção destes, na madeira de algumas espécies, é influenciável pelas condições ambientais que a árvore encontrou no decorrer de seu crescimento. De acordo com Franceschi e Horner (1980) e Nakata (2003), os cristais podem funcionar como defesa contra a herbivoria e atuar na osmorregulação da planta. Lima et al. (2009), estudando o lenho de Enterolobium contortisiliquum, ocorrente em dois ambientes, evidenciaram cristais em todos os indivíduos examinados, porém houve maior predominância de cristais nos indivíduos dos ambientes mais secos.

Visto que, o Stryphnodendron adstringens (barbatimão), exemplo de espécie típica do cerrado, com grande potencial econômico, tanto na indústria farmacêutica, fabricação de tinta para escrever, como de curtimento de couro, em decorrência da grande quantidade de taninos produzidos em suas cascas de até 50\%, e nas suas folhas em torno de 23 a $32 \%$, como comentado por Almeida et al. (2008), e, também, uma vez que, nos tempos atuais, os taninos são de grande interesse não só na indústria de fármacos e de curtimento de couro, os mesmos também podem ser usados para despoluição de água, pois possuem afinidade com metais pesados. Outros tipos de indústrias, interessadas nos taninos e a de produção de adesivos para madeira, tem seu interesse em taninos para produção de um adesivo com menor preço, sem grandes impactos ambientais.

Essa espécie com um enorme potencial econômico já relatado, ligado a produtos florestais não madeireiros (taninos), e à conservação e preservação do bioma cerrado, estudos referentes à química e densidade básica da madeira de S. adstringens (barbatimão), são de vital importância, pois, podem promover maiores conhecimentos relativos à caracterização da espécie e disponibilizar maiores conhecimentos que possibilitam ampliar a gama de utilizações da espécie.

Diante da carência de informações de estudos sobre a química e densidade básica da madeira para as espécies de cerrado, neste estudo, objetivou-se coletar informações sobre a constituição química e densidade básica da madeira de raiz, fuste e galho de Stryphnodendron adstringens (barbatimão).

\section{MATERIAL E MÉTODOS}

\section{1 Área de estudo e coleta do material}

Utilizaram-se, neste estudo, três espécimes de Stryphnodendron adstringens (barbatimão) coletados no município de Lavras, precisamente situada na latitude $21^{\circ} 14^{\prime} 43$ sul, longitude 44 59' 59 oeste, estando a uma altitude média de 919 metros, no sul do estado de Minas Gerais. O clima local, segundo a classificação climática de Köppen, é Cwa, temperado chuvoso (mesotérmico), com inverno seco e verão chuvoso, subtropical, e temperatura do mês mais quente maior que $22^{\circ} \mathrm{C}\left(22,1^{\circ} \mathrm{C}\right.$ em fevereiro).

Foram coletados discos ao longo de todo o comprimento longitudinal em cada espécime, sendo um disco a 0,40 metros abaixo do nível do solo (raiz), cinco discos no fuste $(0,25,50,75$ e $100 \%$ do comprimento total do fuste) e cinco no galho (0, 25, 50, 75 e 100\% do comprimento total dos galhos). Na Figura 1, apresenta-se esquematicamente o critério amostral utilizado.

Os materiais lenhosos da raiz, fuste e galhos foram depositados na Xiloteca da Universidade Federal de Lavras (Tabela 1). Material botânico foi coletado e exsicatas foram preparadas e depositada no Herbário Esal da Universidade Federal de Lavras do departamento de Ecologia da Universidade Federal de Lavras MG (Tabela 1).

\subsection{Preparo do material para análises físicas e químicas}

Foram retirados dos discos, cunhas opostas para análises de densidade básica e o restante do material foi utilizado para análises químicas. Para as análises químicas, os materiais de todos os espécimes e de cada posição, foram processados, e convertidos em serragem. Esta por sua vez foi classificada em função da granulometria com auxílio de um conjunto de peneiras com porosidade de 40 e 60 mesh. A serragem retida entre as duas peneiras foi acondicionada em câmara climática a $20 \pm 2{ }^{\circ} \mathrm{C}$ e $60 \pm 5 \%$ UR, para estabilização da sua umidade e posterior utilização nas analises químicas.

\subsection{Análises químicas}

As análises químicas foram realizadas no Laboratório de Tecnologia da Madeira da UFLA, conforme as normas descritas na Tabela 2, para teor de extrativos totais, cinzas e lignina. O teor de holocelulose foi obtido por diferença. Foram feitas análises individuais por disco e posterior média dos valores para representar a respectiva posição do vegetal.

Cerne, Lavras, v. 18, n. 1, p. 59-66, jan./mar. 2012 


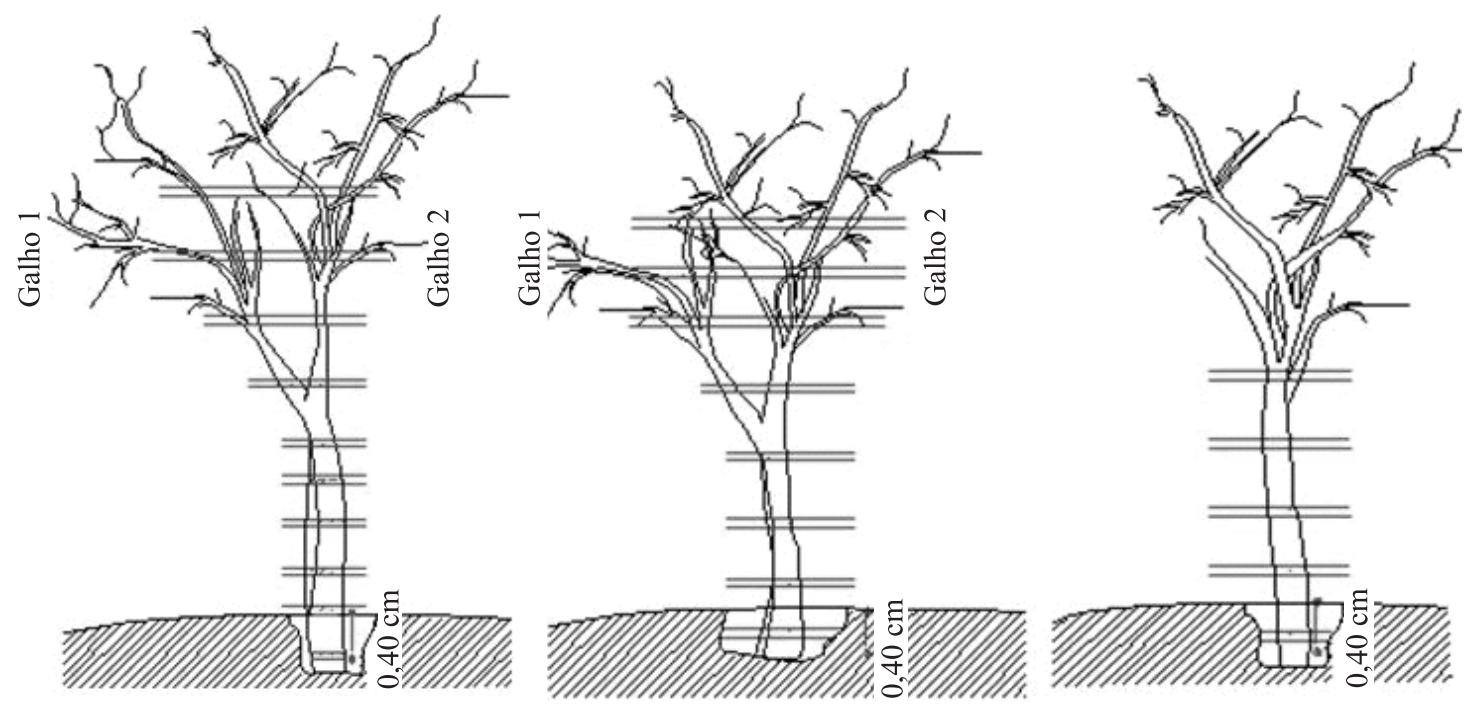

Figura 1 - Diagrama de perfil mostrando as principais ramificações dos três espécimes de (Stryphnodendron adstringens (barbatimão). Os traços indicam o local amostrado.

Figure 1 - Profile diagram of the main branching parts of three specimens of Stryphnodendron adstringens (barbatimão). Bars indicate the sampled area.

Tabela 1 - Informações sobre os espécimes coletados de Stryphnodendron adstringens (barbatimão).

Table 1 - Information on the sampled specimens of Stryphnodendron adstringens (barbatimão).

\begin{tabular}{lcccccccc}
\hline Coletor & Espécime & Posição & $\begin{array}{c}\mathrm{N}^{\circ} \text { Herbário } \\
\text { Esal }\end{array}$ & $\begin{array}{c}\mathrm{N}^{\circ} \\
\text { Xiloteca }\end{array}$ & $\begin{array}{c}\text { Altura } \\
(\mathrm{m})\end{array}$ & $\begin{array}{c}\text { DAP } \\
(\mathrm{cm})\end{array}$ & $\begin{array}{c}\text { Diâm. raiz } \\
(\mathrm{cm})\end{array}$ & $\begin{array}{c}\text { Diâm. galho } \\
(\mathrm{cm})\end{array}$ \\
\hline Goulart, S. L & 1 & $\mathrm{R}$ & - & 268 & - & - & 14,5 & - \\
Goulart, S. L & 1 & $\mathrm{~F}$ & 4789 & 269 & 5,11 & 13,5 & - & - \\
Goulart, S. L & 1 & $\mathrm{G}$ & - & 270 & - & - & - & - \\
Goulart, S. L & 2 & $\mathrm{R}$ & - & 271 & - & - & - & - \\
Goulart, S. L & 2 & $\mathrm{~F}$ & 4779 & 272 & 3,4 & 10 & - & - \\
Goulart, S. L & 2 & $\mathrm{G}$ & - & 273 & - & - & - & - \\
Goulart, S. L & 3 & $\mathrm{R}$ & - & 274 & - & - & 16,5 & - \\
Goulart, S. L & 3 & $\mathrm{~F}$ & 4776 & 275 & 4,62 & 14,5 & - \\
\hline
\end{tabular}

$\mathrm{N}^{\mathbf{o}}=$ número. $\mathrm{DAP}=$ Diâmetro a altura do peito $(1,30 \mathrm{~m})$. Diâm = diâmetro. $\mathrm{R}=$ raiz. $\mathrm{F}=$ fuste. $\mathrm{G}=$ galho.

Tabela 2 - Análise química com as respectivas normas.

Table 2 - Chemical analysis with relevant ABTCP standards.

\begin{tabular}{lc}
\hline Análise & Norma \\
\hline Teor de extrativos totais & ABTCP m 3/69 \\
Teor de cinzas & ABTCP m 11/77 \\
Teor de lignina (klason) & ABTCP m 10/71 \\
Teor de holocelulose & Obtido por diferença \\
\hline
\end{tabular}

ABTCP = Associação brasileira de técnica de celulose e papel.

\subsection{Análises físicas}

As densidades foram realizadas no Laboratório de Tecnologia da Madeira da UFLA, empregando-se a norma NBR 7190 (ASSOCIAÇÃO BRASILEIRA DE NORMAS TÉCNICAS - ABNT, 1997).

\subsection{Análises estatísticas}

Para análise dos dados, utilizou-se software SISVAR Sistema de Análise de Variância Para Dados Balanceados

Cerne, Lavras, v. 18, n. 1, p. 59-66, jan./mar. 2012 
(DEX-UFLA). Foram feitas análise de variância em DIC e teste de média (Tukey) a 0,05 de significância.

\section{RESULTADOS E DISCUSSÃO}

\subsection{Caracterização química}

Por meio das análises realizadas, foi possível comparar o teor de extrativos, cinzas, lignina e de holocelulose entre raiz, fuste e galho, dos espécimes estudados, (Tabela 3 e Figuras 2 e 3). Os valores encontrados para holocelulose, tanto na raiz quanto no fuste e galho, superaram a faixa de composição da madeira proposta por Klock et al. (2005). Como pode ser observado na (Figura 2), não houve diferença estatística para holocelulose entre as posições estudadas, houve apenas uma tendência de maiores teores nos galhos, esses resultados estão de acordo com Zobel e Buijtenen (1989), que afirmam que a madeira juvenil apresenta maiores teores em holocelulose, quando comparada com a do lenho adulto. Silva et al. (2005), estudando madeira de Eucalyptus grandis, verificaram que árvores mais jovens e que a amostragem das partes mais superiores do tronco apresentaram maiores teores de holocelulose, os autores relatam ainda que a variabilidade interna das árvores, no sentido longitudinal, influiu mais decisivamente na composição química da madeira que a variação da idade. Na Figura 2, apresentam-se, graficamente, os dados médios obtidos para o teor de holocelulose e lignina.

Os teores de lignina, no geral, apresentaram-se em torno de 22,5 \%, para o galho, 23,7\% para o fuste e 24,6 $\%$ para a raiz. Esses valores são considerados baixos para madeiras de dicotiledôneas (KLOCK et al., 2005). Houve diferença significativa para o teor de lignina entre as posições (Figura 2). De acordo com Bodig e Jayne (1982), existe grande variabilidade quantitativa dos componentes químicos na madeira de uma mesma espécie e, inclusive, no mesmo indivíduo. Pettersen (1984) esclarece que esse fato, pode ocorrer em decorrência do local das partes da árvore em que a amostra foi retirada, assim como as condições ambientais a que a mesma esteve submetida. Santana e Okino (2007) comentam que essa variação pode ser esclarecida também pela idade das árvores coletadas. Os espécimes de S.adstringens (barbatimão) são de ambiente de cerrado, a idade destas varia consideravelmente, mesmo quando se trata de uma área pequena.

Para os teores de extrativos, não houve diferenças estáticas significativas entre as posições estudadas (Figura 3), porém, a raiz apresentou uma tendência de maiores teores desses componentes. Os valores encontrados de
Tabela 3 - Resumo da Anava de S.adstringens (barbatimão).

Table 3 - Anava summary for S.adstringens (barbatimão).

\begin{tabular}{|c|c|c|c|c|c|}
\hline \multirow{2}{*}{ FV } & \multirow{2}{*}{ GL } & \multicolumn{2}{|c|}{ Física } & \multicolumn{2}{|c|}{ Química } \\
\hline & & DB & EXT & LIG & HOL \\
\hline Posições & 5 & $0,0001^{\mathrm{ns}}$ & $39,94^{\mathrm{ns}}$ & $2,79 *$ & $61,19^{\text {ns }}$ \\
\hline \multirow[t]{2}{*}{ Erro } & 2 & 0,001 & 37,44 & 2,26 & 36,75 \\
\hline & $\mathrm{CV}_{\text {exp }}(\%)$ & 5,15 & 26,90 & 2,17 & 11,34 \\
\hline
\end{tabular}

* Significativo a 0,05 de significância

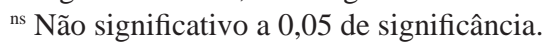

DB = densidade básica; EXT = extrativo; $\mathrm{LIG}$ = lignina; $\mathrm{HOL}=$ holocelulose.
Holocelulose (\%)

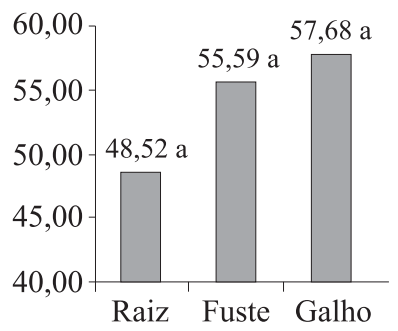

Lignina (\%)

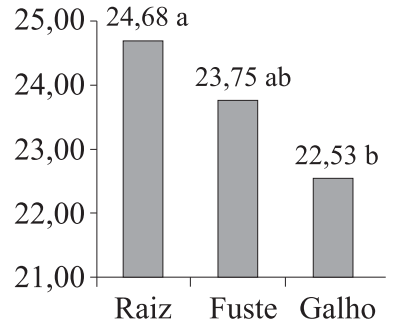

Figura 2 - Teores de holocelulose e lignina, dos lenhos de raiz, fuste e galho de S.adstringens (barbatimão).

Figure 2 - Holocellulose and lignin contents in the root, stem and branch portions of S.adstringens (barbatimão).
Extrativos (\%)

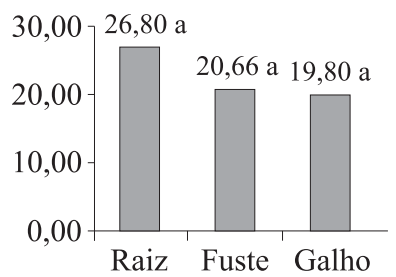

Cinzas $(\%)$

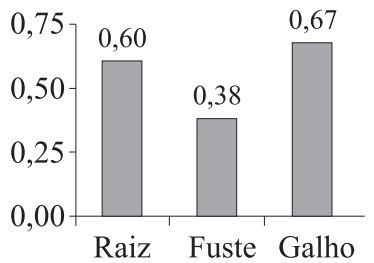

Figura 3 - Teores de extrativos e cinzas, dos lenhos de raiz, fuste e galho de S.adstringens (barbatimão).

Figure 3 - Extractive and ash contents in the root, stem and branch portions of S.adstringens (barbatimão).

extrativos, tanto para o lenho de raiz de $26,8 \%$, fuste $20,6 \%$, e galho $19,7 \%$, são considerados, no geral, valores altos, segundo Klock et al. (2005) e Tsoumis (1991). De acordo com Buchanan (1981) e Oliveira et al. (2005), os extrativos mesmo quando presentes em pequenas proporções, estão entre os principais responsáveis pela resistência da madeira ao ataque de fungos e insetos.

Cerne, Lavras, v. 18, n. 1, p. 59-66, jan./mar. 2012 
O fato da maior quantidade de extrativos, na raiz da espécie em estudo, pode estar relacionado a uma maior proteção do lenho da raiz ao ataque desses organismos. A menor quantidade de extrativos no galho pode estar relacionada ao fato de que a madeira do galho está na fase juvenil, estando de acordo com o que foi relatado por Silva (2002) e Trugilho et al. (1996), em que os teores de extrativos totais na madeira tendem a ser menores em madeiras mais jovens, em razão da menor proporção em madeira adulta. Segundo Zobel e Talbert (1984) há maior proporção de madeira juvenil no topo da árvore e menor é a proporção da mesma na base. Na Figura 3, apresentam-se, graficamente, os valores médios dos teores de extrativos totais e cinzas.

Para o teor de minerais, o galho apresentou o maior valor comparado às demais posições (Figura 3). Esses componentes são, geralmente, representados por oxalatos e sílica e a proporção destes na madeira de algumas espécies é influenciável pelas condições ambientais que a árvore encontrou no decorrer de seu crescimento (BUCHANAM, 1981).

\subsection{Análise física}

Estatisticamente não houve diferença para os valores de densidade básica, e os mesmo, mostraram-se médios, em torno de $0,459 \mathrm{~g} / \mathrm{cm}^{3}$ para raiz, $0,468 \mathrm{~g} / \mathrm{cm}^{3}$ para fuste e o galho com $0,452 \mathrm{~g} / \mathrm{cm}^{3}$ (Figura 4), estando de acordo com os resultados obtidos por Détienne e Jacquet (1983), para o gênero Stryphnodendron entre 0,45 e 0,65 $\mathrm{g} / \mathrm{cm}^{3}$ e Souza et al. (2008) para a espécie $S$. adstringens (barbatimão), em diferentes regiões do estado de Minas Gerais, entre 0,265 a $0,605 \mathrm{~g} / \mathrm{cm}^{3}$ e, segundo os autores, os fatores ambientais influenciaram nas características da densidade básica neste estudo. Vale et al. (2002) comentam que a densidade básica da madeira de espécies do cerrado apresenta uma variação de $0,20 \mathrm{~g} / \mathrm{cm}^{3}$ a $0,78 \mathrm{~g} / \mathrm{cm}^{3}$.

Observou-se uma leve tendência de maior valor da densidade para o fuste e menor para o galho. Nos estudos de Vale et al. (1992), com comportamento da densidade básica da madeira de espécies nativas do cerrado, Vochysia thyrsoidea (Gomeira), Sclerolobium paniculatum (Carvoeiro) e Pterodon pubescens (Sucupira branca), encontraram valores de 0,54, 0,70 e $0,75 \mathrm{~g} / \mathrm{cm}^{3}$, respectivamente. Os autores comentam, ainda, que as espécies apresentaram comportamento diferenciado para a densidade básica da madeira ao longo do tronco e no sentido medula-casca; e que a densidade da gomeira e da sucupira branca apresentou-se constante da base para o topo, já, na do carvoeiro houve tendência na sua redução,

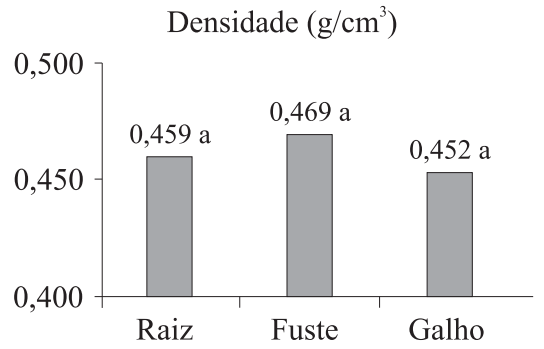

Figura 4 - Valores de densidade básica dos lenhos de raiz, fuste e galho de $S$. Adstringens (barbatimão).

Figure 4 - Basic density values in the root, stem and branch portions of S. Adstringens (barbatimão).

em função da altura. Oliveira et al. (2008) relatam em seus estudos que o carvoeiro (Sclerolobium paniculatum) apresentou densidade básica média de $0,52 \mathrm{~g} / \mathrm{cm}^{3}$.

$\mathrm{O}$ fato de a madeira do galho de S.adstringens ter apresentado uma tendência para menor valor, pode estar relacionado ao estado juvenil que o mesmo se encontra. Silva et al. (2009), em estudos de Caesalpinia pyramidalis, comprovaram que a mesma, apresentou no alburno e no cerne do tronco e dos galhos, densidade próxima ou maior que $1,0 \mathrm{~g} / \mathrm{cm}^{3}$. E, segundo os autores, os galhos apresentam potencial semelhante ao do tronco para a densidade básica.

\section{CONCLUSÕES}

Os valores encontrados para holocelulose na raiz, no fuste e galho, superaram a faixa de composição química das madeiras tropicais.

Estatisticamente, entre as posições estudadas, não houve diferença para o teor de holocelulose.

Os teores de ligninas, no geral, apresentaram-se baixos para madeiras de dicotiledôneas.

Em relação aos extrativos, não houve diferenças estatísticas significativas entre as posições estudadas, porém a raiz apresentou o maior teor. No geral, foram considerados com valores altos.

Observou-se uma leve tendência de maior valor em densidade básica para o fuste e menor para o galho. Os valores encontrados para a densidade básica, no geral, estão dentro dos valores encontrados para as espécies de cerrado.

\section{AGRADECIMENTOS}

À FAPEMIG e ao CNPq, pelo apoio financeiro para realização deste trabalho e à Ciência e Tecnologia da Madeira/UFLA.

Cerne, Lavras, v. 18, n. 1, p. 59-66, jan./mar. 2012 


\section{REFERÊNCIAS}

ALMEIDA, N. F. de; MORI, F. A.; GOULART, S. L.; MENDES, L. M.; RIBEIRO, A. O. Rendimentos em taninos das folhas de barbatimão [Stryphnodendron adstringens] em diferentes períodos de coleta visando a produção de adesivo para a maderia. In: ENCONTRO BRASILEIRO EM MADEIRA E ESTRUTURAS DE MADEIRA, 11., 2008, Londrina. Anais... Londrina, 2008. p. 5-8.

\section{ASSOCIAÇÃO BRASILEIRA DE NORMAS TÉCNICAS.}

NBR 7190: projetos de estruturas de madeira. Rio de Janeiro, 1997. $107 \mathrm{p}$.

BODIG, J.; JAYNE, B. A. Mechanics of wood and wood composites. New York: V. N. Reinhold, 1982. 712 p.

BUCHANAN, M. A. Extraneous components of wood: the chemistry of wood. New York: R. Krieger, 1981. 689 p.

CHIMELLO, J. Anotações sobre anatomia e identificações de madeiras. São Paulo: IPT, 1980. Apostila.

DÉTIENNE, P.; JAQUET, P. Atlas d'identification des bois de l'Amazonie et des régions voisines. Paris: Centre Technique Forestier Tropical, 1983. 641 p.

FRANCESCHI, V. R.; HORNER, H. T. Calcium oxalate crystals in plant. The Botanical Review, Bronx, v. 46, p. 361427, 1980.

FREDDO, A. Elementos minerais em madeira de eucaliptos e acácia negra e sua influência na indústria de celulose kraft branqueada. 1997. 69 f. Dissertação (Mestrado em Engenharia Florestal) - Universidade Federal de Santa Maria, Santa Maria, 1997.

KLOCK, U.; MUÑIZ, G.; HERNANDEZ, J.; ANDRADE, A. Química da madeira. Curitiba: UFPR, 2005. 86 p. Apostila.

LEPAGE, E. S.; OLIVEIRA, A. M. F.; LELIS, A. T.; LOPEZ, G. A. C.; CHIMELLO, J. P.; OLIVEIRA, L. C. S.; CAÑEDO, M. D.; CAVALCANTE, M. S.; IELO, P. K. Y.; ZANOTTO, P. A.; MILANO, S. Manual de preservação de madeiras. 2. ed. São Paulo: IPT, 1986. 708 p.

LIMA, R. S. de; OLIVEIRA, P. L. de; RODRIGUES, L. R. Anatomia do lenho de Enterolobium contortisiliquum (Vell.)
Morong (Leguminosae-Mimosoideae) ocorrente em dois ambientes. Revista Brasileira de Botânica, São Paulo, v. 32, n. 2, p. 361-374, abr./jun. 2009.

MARCHESAN, R.; MATTOS, P. P. de; BORTOLI, C. de; ROSOT, N. C. Caracterização física, química e anatômica da madeira de Ocotea porosa (Nees \& C. Mart.) barroso. Colombo: Embrapa, 2006. 3 p.

MATTOS, P. P. de; PEREIRA, J. C. D.; SCHAITZA, E. G.; BAGGIO, A. J. Caracterização física, química e anatômica da madeira de Terminalia ivorensis. Colombo: Embrapa, 1999.

MENDES, L. M.; SILVA, J. R. M. da; TRUGILHO, P. F.; LOPES, G. de A. Anatomia e características físico-quimícas da madeira de Zanthoxylum tingoassuiba St. Hil. de ocorrência na região de Lavras/MG. Lavras: UFLA, 2002.

METCALFE, C. R.; CHALK, L. Anatomy of the dicotiledons, wood structure and conclusion of the general introduction. Oxford: Clarendon, 1983. 297 p.

MORAIS, S. A. L. de; NASCIMENTO, E. A. do; MELO, D. C. de. Análise da madeira de Pinus oocarpa parte I: estudo dos constituintes macromoleculares e extrativos voláteis. Revista Árvore, Viçosa, v. 29, n. 3, p. 461-470, 2005.

NAKATA, P. A. Advances in our understanding of calcium oxalate crystal formation and function in plant. Plant Science, Shannon, v. 164, p. 901-909, 2003.

OLIVEIRA, I. da R. M. de; VALE, A. T. do; MELO, J. T. de; COSTA, A. F. da; GONÇALEZ, J. C. Biomassa e características madeira de Sclerolobium paniculatum, cultivado em diferentes níveis de adubação. Cerne, Lavras, v. 14, n. 4, p. 351-357, out./dez. 2008.

OLIVEIRA, J. T. da S.; SOUZA, L. C.; DELLA LUCIA, R. M.; SOUZA JÚNIOR, W. P. de. Influência dos extrativos na resistência ao apodrecimento de seis espécies de madeira. Revista Árvore, Viçosa, v. 29, n. 5, p. 819-826, set./out. 2005.

PANSHIN, A. J.; ZEEUW, C. de. Textbook of wood technology. 3. ed. New York: McGraw Hill, 1980. 722 p.

PAULA, J. E. Madeiras da caatinga úteis para produção de energia. Pesquisa Agropecuária Brasileira, Brasília, v. 28, n. 1, p. 153-165, jan. 1993.

Cerne, Lavras, v. 18, n. 1, p. 59-66, jan./mar. 2012 
PETTERSEN, R. C. The chemical composition of wood. In: ROWELL, R. (Ed.). The chemistry of solid wood. Washington: American Chemical Society, 1984. p. 54-126.

ROWELL, W. C. (Ed.). Handbook of wood chemistry and wood composites. Boca Raton: CRC, 2005. 487 p.

SANTANA, M. A. E.; OKINO, E. Y. A. Chemical composition of 36 Brazilian Amazon forest wood species. Holzforschung, Berlin, v. 61, p. 468-477, 2007.

SEVERO, E. T. D.; CALONEGO, F. W.; SANSÍGOLO, C. A. Composição química da madeira de Eucalyptus citriodora em função das direções estruturais. Silva Lusitana, Lisboa, v. 14, n. 1, p. 113-126, 2006.

SILVA, G. J.; CAMPELO JÚNIOR, J. H.; BRAWERS, L. R.; DURAN, J. A. R. Avaliação de plantas adultas de espécies arbóreas do cerrado em função do clima. Agricultura Tropical, Cuiabá, v. 8, p. 43-46, 2004.

SILVA, J. de C. Caracterização da madeira de Eucaliptus grandis Hill ex. Maiden, de diferentes idades, visando a sua utilização na indústria moveleira. 2002. $160 \mathrm{f}$. Tese (Doutorado em Tecnologia e Utilização de Produtos Florestais) - Universidade Federal do Paraná, Curitiba, 2002.

SILVA, J. de C.; MATOS, J. L. M. de; OLIVEIRA, J. T. da S.; EVANGELISTA, W. V. Influência da idade e da posição ao longo do tronco na composição química da madeira de Eucalyptus grandis Hill ex. Maiden1. Revista Árvore, Viçosa, v. 29, n. 3, p. 455-460, 2005.

SILVA, L. B. da; SANTOS, F. de A. R. dos; GASSON, P.; CUTLER, D. Anatomia e densidade básica da madeira de
Caesalpinia pyramidalis Tul. (Fabaceae), espécie endêmica da caatinga do Nordeste do Brasil. Acta Botânica Brasílica, Porto Alegre, v. 23, n. 2, p. 436-445, 2009.

SOUZA, F. N. de; RUFINI, A. L.; SCOLFORO, J. R. S. Análise da variação da densidade para Anadenanthera colubrina (ANGICO), Stryphnodendron adstringens (BARBATIMÃO) e Kielmeyera coriacea (PAU SANTO) no estado de Minas Gerais. In: ENCONTRO BRASILEIRO EM MADEIRA E ESTRUTURAS DE MADEIRA, 11., 2008, Londrina. Anais... Londrina, 2008. CD-ROM.

TRUGILHO, P. F.; LIMA, J. T.; MENDES, L. M. Influência da idade nas caracterís-ticas físico-mecânicas e anatômicas da madeira de Eucalyptus saligna. Cerne, Lavras, v. 2, n. 1, p. 97-111, jan./jun. 1996.

TRUGILHO, P. F.; LIMA, J. T.; MORI, F. A. Correlação canônica das características químicas e físicas de clones de Eucalyptus grandis e Eucalyptus saligna. Cerne, Lavras, v. 9, n. 1, p. 66-80, jan./mar. 2003.

TSOUMIS, G. Science and technology of wood: structure, properties, utilization. New York: V. N. Reinold, 1991. 494 p.

VALE, A. T.; MARTINS, I. S.; ARAÚJO, W. O. Estudo da densidade básica de três espécies do cerrado. Revista Árvore, Viçosa, v. 16, n. 2, p. 209-217, 1992.

ZOBEL, B. J.; BUIJTENEN, J. P. van. Wood variation: its causes and control. New York: Springer-Verlag, 1989. 363 p.

ZOBEL, B. J.; TALBERT, J. Applied forest tree improvement. New York: Wiley, 1984. 511 p.

Cerne, Lavras, v. 18, n. 1, p. 59-66, jan./mar. 2012 